CPT-2000/P.4003.

\title{
Positivity constraints and flavor dependence of higher twists
}

\author{
J. Soffer 由 \\ Centre de Physique Théorique - CNRS - Luminy, \\ Case 907 F-13288 Marseille Cedex 9 - France \\ and O. V. Teryaev? \\ Bogoliubov Laboratory of Theoretical Physics, \\ Joint Institute for Nuclear Research, Dubna, 141980, Russia
}

\begin{abstract}
The positivity bound for the transverse asymmetry $A_{2}$ may be improved and applied for each quark flavor separately. We use it to test the consistency of twist-two approximation for the transverse spin-dependent $\left(g_{T}\right)$ and the longitudinal spin-averaged $\left(F_{L}\right)$ structure functions. While it is satisfied in the case of $u$ quarks, it might be violated for $d$ quarks in a region of moderate $x$ because of its negative polarization. We attribute this inconsistency to a stronger twist-three contribution, whose existence found by the QCD sum rule method is a long-standing puzzle.
\end{abstract}

Positivity is playing a very important role in constraining various spindependent observables, in particular by providing a bound for the transverse asymmetry in polarized Deep Inelastic Scattering (DIS). It is a well-known condition established long time ago [1] and based on an extensive study by Doncel and de Rafael [2], written in the form

$$
\left|A_{2}\right| \leq \sqrt{R}
$$

where $A_{2}$ is the usual transverse asymmetry and $R=\sigma_{L} / \sigma_{T}$ is the standard ratio in DIS of the cross section of longitudinally to transversely polarized off-shell photons. It reflects a non-trivial positivity condition one has on the

\footnotetext{
${ }^{1}$ E-mail: soffer@cpt.univ-mrs.fr

2 E-mail: teryaev@thsun1.jinr.ru
} 
photon-nucleon helicity amplitudes. By substituting photons for gluons, we found earlier [3], that the similar bound holds for the various matrix elements for longitudinal gluons in a nucleon [4]

$$
\left|\Delta G_{T}(x)\right| \leq \sqrt{1 / 2 G(x) G_{L}(x)} .
$$

However, this bound can be rederived in line with the positivity bound in the quark case, known as Soffer inequality [5],

$$
\left|h_{1}(x)\right| \leq q_{+}(x)=\frac{1}{2}[q(x)+\Delta q(x)],
$$

by making the substitution in Eq.(2), $G(x) \rightarrow G_{+}(x)=\frac{1}{2}[G(x)+\Delta G(x)]$, and providing a stronger restriction, especially when the gluon helicity distribution $\Delta G(x)$ is small or even negative. Coming back to the photon case, if $A_{1}$ denotes the asymmetry with longitudinally polarized nucleon, we are led to

$$
\left|A_{2}\right| \leq \sqrt{R\left(1+A_{1}\right) / 2}
$$

a stronger bound than Eq.(1). In the present paper we will show that this is really the case, using a transparent physical approach, and generalize it to consider each quark flavor separately. We show that this leads to sensitive tests for parton distribution and sometimes give a hint about higher twist terms. We will also comment on, why, we think the weaker bound was used up to now.

We start with the following expressions for the various photon-nucleon cross-sections in terms of the matrix elements describing the transition from the state $\mid H, h>$ of a nucleon with helicity $h$ and a photon with helicity $H$, to the unobserved state $\mid X>$

$$
\begin{array}{r}
\sigma_{T}^{ \pm}=\sum_{X}|<+1 / 2, \pm 1| X>\left.\right|^{2}, \\
\sigma_{L}=\sum_{X}|<+1 / 2,0| X>\left.\right|^{2}=\sum_{X}|<-1 / 2,0| X>\left.\right|^{2}, \\
\sigma_{L T}=2 \operatorname{Re} \sum_{X}<+1 / 2,+1|X><-1 / 2,0| X>.
\end{array}
$$

Note that while longitudinal $\sigma_{L}$ and transverse $\sigma_{T}=\sigma_{T}^{+}+\sigma_{T}^{-}$cross-sections are symmetric with respect to the reverse of the nucleon and photon helicities, 
this is not the case for the interference term $\sigma_{L T}$. The reason is very simple: the opposite helicities of photon and nucleon correspond to their spins parallel, so that the angular momentum of the state $|X\rangle$ has its maximum value $3 / 2$. The amplitude, which could possibly interfere with it to produce the transverse asymmetry, should have the same total angular momentum of the state $|X\rangle$. This is however impossible, as the flip of the one of the helicities would require another one to exceed its maximal possible value, in order to keep the angular momentum of $|X\rangle$ the same. Therefore the interference, responsible for $A_{2}$, does not occur. This is quite a general reason, for the occurence of the + helicity configurations in all the cases considered above.

We are now ready to write down the Cauchy-Schwarz inequality as

$$
\sum_{X}|<+1 / 2,+1| X> \pm a<-1 / 2,0|X>|^{2} \geq 0
$$

where $a$ is a positive real number. By making use of the definitions (5) and after the standard minimization with respect to the choice of $a$, one immediately arrives at

$$
\left|\sigma_{L T}\right| \leq \sqrt{\sigma_{L} \sigma_{T}^{+}}
$$

leading directly to (4).

The next important step is to apply this method for each quark flavor separately. This may be achieved by considering a fictitious "photon" coupled to only one flavor. In other words, this is just the consideration of positivity [2] for each flavor contribution to the structure function $W^{\mu \nu}$, i.e.

$$
W_{f}^{\mu \nu} \epsilon_{\mu} \epsilon_{\nu}^{*}>0 \text {. }
$$

So, if we have the following definition

$$
\sigma_{i}=\sum_{f} e_{f}^{2} \sigma_{i}^{f}(i=L, T, L T),
$$

positivity implies, that

$$
\left|\sigma_{L T}^{f}\right| \leq \sqrt{\sigma_{L}^{f} \sigma_{T}^{+f}}
$$


or analogously to eq. (卌)

$$
\left|A_{2}^{f}\right| \leq \sqrt{R^{f}\left(1+A_{1}^{f}\right) / 2} .
$$

Note of course that all the kinematic factors correspond to the nucleon, so that, say, no quark masses will appear.

We are now going to make use of the fact that these inequalities involve terms of different twists which may be used to put some constraints on their relative size and $Q^{2}$-evolution [6]. The improved bound at hand is a practical tool to start such an investigation. Let us consider, as a first step, the twisttwo approximation at Born level for both $\sigma_{L}^{f}$ and $\sigma_{L T}^{f}$. This means that we will take the Wandzura-Wilczek (WW) [7] approximation for $\sigma_{L T}^{f}$ and the target mass approximation [8] for $\sigma_{L}^{f}$.

Consequently, one should keep only the twist-two part of the transverse spin structure functin $g_{T}(x)=g_{1}(x)+g_{2}(x)$ for each quark flavor $f$

$$
g_{T}^{f}(x)=g_{T}^{f, W W}(x)+\bar{g}_{2}^{f}(x)
$$

with

$$
g_{T}^{f, W W}(x)=\int_{x}^{1} \frac{d z \Delta f(z)}{z},
$$

represented by the first term in the r.h.s., disregarding the twist-three part, which, in this case, is entirely provided by the twist-three part of structure function $g_{2}$. At the same time, one should disregard the twist-four part of the longitudinal structure function $F_{L}$, as well as the twist-two part coming from the radiative corrections s. The quantities $A_{2}^{f}$ and $R^{f}$ should then be effectively replaced by

$$
\begin{gathered}
A_{2}^{f} \rightarrow \frac{2 M}{Q} x g_{T}(x) \rightarrow \int_{x}^{1} \frac{d z \Delta f(z)}{z} ; \\
R^{f} \rightarrow \frac{F_{L}^{f}(x)}{F_{2}^{f}(x)} \rightarrow \frac{4 M^{2} x^{2}}{Q^{2}} \frac{\int_{x}^{1} \frac{d z f(z)}{z}}{f(x)} .
\end{gathered}
$$

\footnotetext{
${ }^{3}$ Note that the gluon distribution will enter the constraint for each quark flavor at next-to-leading order.
} 
Then positivity requires that

$$
\left|\int_{x}^{1} \frac{d z \Delta f(z)}{z}\right| \leq \sqrt{f_{+}(x) \cdot \int_{x}^{1} \frac{d z f(z)}{z}}=\sqrt{\frac{f(x)+\Delta f(x)}{2} \cdot \int_{x}^{1} \frac{d z f(z)}{z}} .
$$

We checked this bound and found that it is strongly dependent on the parametrization one uses for the quark distributions. Especially sensitive is the case of $d$ quark, because of its negative polarization. While the inequality is satisfied by the GRSV [9] distributions, the GS [10] distributions exhibit a tiny violation of the inequality in the case of $d$-quark for $0.4 \leq x \leq 0.8$ (see Fig.1), but not for the $u$-quark (Fig. 2). We also anticipate a violation in the case of the strange quark, whose polarization, although losely known, is expected to be negative.

We make two general conclusions from such a controversial situation. First, the generalized bound (15) is a new sensitive test for the quark distributions. Second, the precise experimental data in the large $x$ region, which should be obtained at CEBAF, will be of great importance to resolve the controversy,

If the violation of the constraint is confirmed, it will be considered as an indication of the inconsistency of the twist-two Born approximation and the necessity to account for higher orders of perturbation theory and higher twists. The simplest way to do this would be to assume a non-zero twist-three $\bar{g}_{2}^{f}$ (see eq. (12)).

This guess is also justified by the fact, that the constraint (11) may be, in principle, violated by WW approximation regardless of the approximation one uses for $F_{L}$. To see that, let us consider a parton distribution, for which the quark polarization is large and negative in the region of moderate $x$. Then, on the one side, $g_{T}^{W W}$ should be large. At the same time, the quantity $f_{+}$is small and the bound is violated. Physically, this can be interpreted in the following way. The WW approximation may be obtained [11] by accounting for an intrinsic transverse momentum $k_{T}$ of the polarized quark. Violation of positivity tells, that such a $k_{T}$ kick, in general, may result in the hadronic remnant $|X\rangle$, not being physical. The approximation for $\sigma_{L}$ is actually not very important, since the violation of positivity comes from the smallness of the amplitude $\langle+1 / 2,+1 \mid X\rangle$, which does not enter in the definition of $\sigma_{L}$. The key ingredient of WW, resulting in this property is the expression of $g_{T}$ entirely in terms of $g_{1}$. Therefore, the twist-three contribution is, in general, a necessary ingredient to restore positivity. 


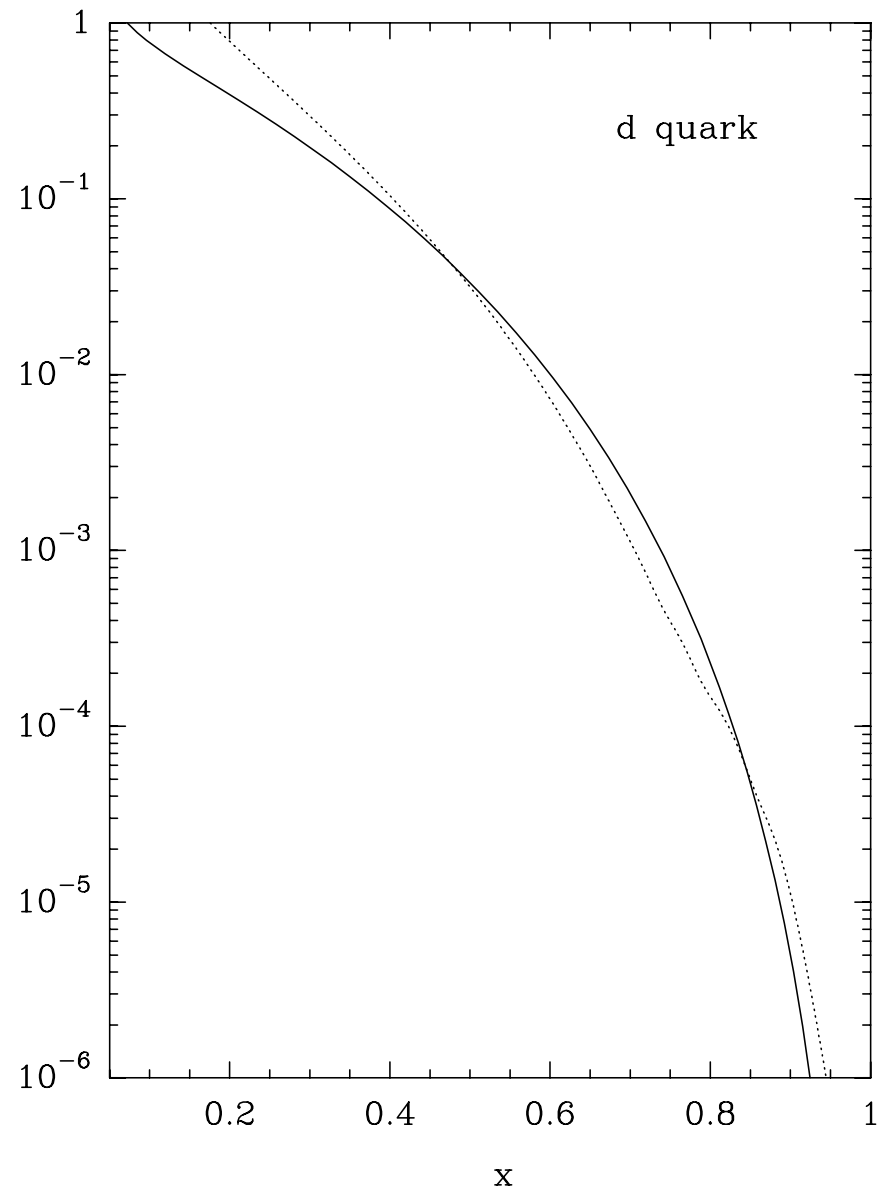

Figure 1: Test of the positivity bound using the GS distribution for $d$ quark at $Q^{2}=1 \mathrm{GeV}^{2}$. The solid curve corresponds to the l.h.s. of eq (15) and the dotted curve to the r.h.s. 


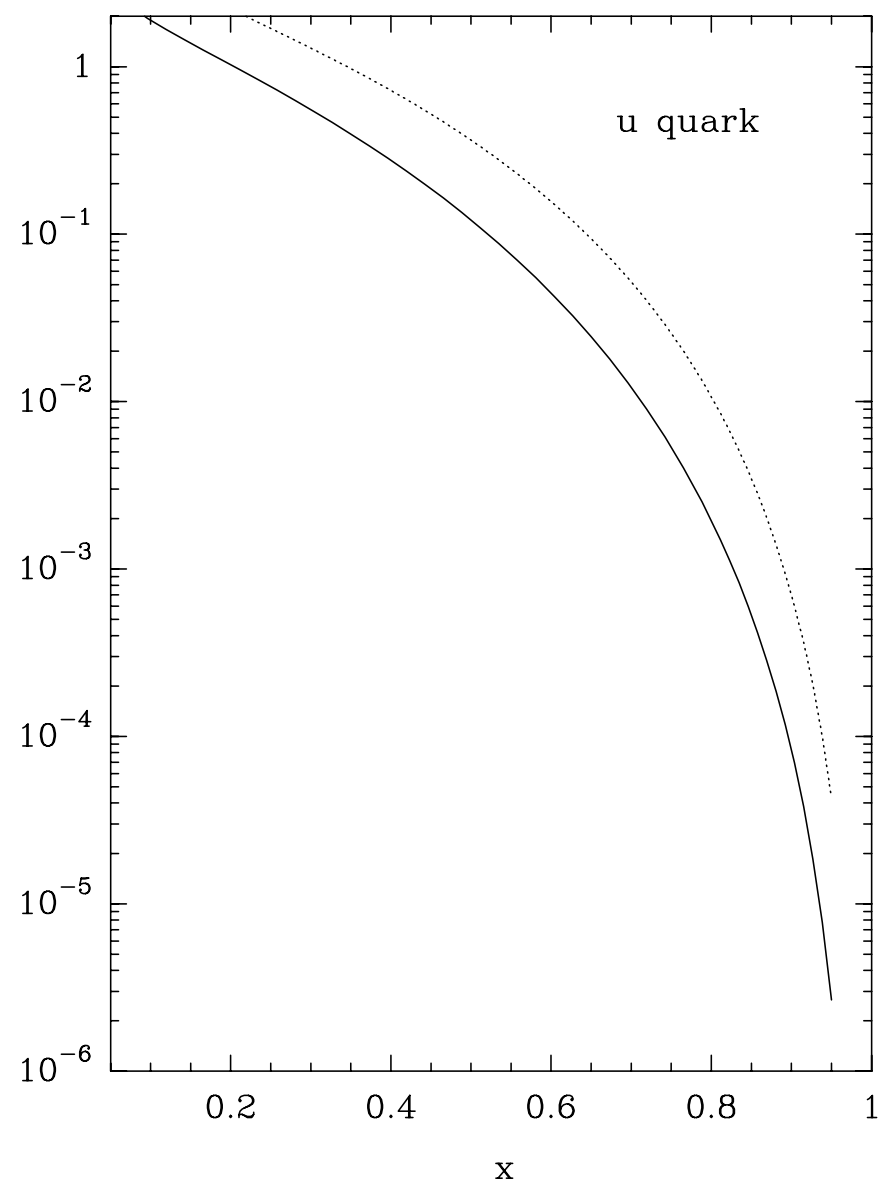

Figure 2: Same as Fig. 四, but for $u$ quark. 
The $d$ quark, which has a negative polarization makes such a qualitative arguments more profound. This may lead to a long-awaited qualitative reason for the strong flavor dependence of twist-three contributions found by QCD sum rule method [12, 13. It is in fact resulting in an order of magnitude larger value of its third moment

$$
\int_{0}^{1} d x x^{2} \bar{g}_{2}^{f}(x) \equiv \frac{1}{3} d_{2}^{f}
$$

for neutron with respect to proton, and consequently for $d$ quark with respect to $u$ quark.

Although it may be too early to use the current parametrizations for quantitative estimates, let us discuss some relevant implications. Note first, that because the WW contribution is negative for $d$ quark, the twist-three part should be positive to restore the validity of the constraint. At the same time, to make any statement about the sign of $d_{2}$ one should take into account, that $\bar{g}_{2}$ should change sign due to the Burkhardt-Cottingham sum rule (whose possible violations cannot appear in the framework of operatorproduct expansion, being the basis of quantitative analysis of twist-three effects) [14]. Moreover, if the sea quark contribution is negligible, the sign changes should be even more dramatic due to the so-called Efremov-LeaderTeryaev sum rule 15. Surprizingly enough, the simple model [1], based on these two sum rules predicts the existence of a maximum of $\bar{g}_{2}$ at $x \sim 0.6$, which is not far from the region where positivity at twist-two level is violated. Moreover, the sign of the third moment happens to be opposite to the sign of $\bar{g}_{2}$ in this point, so that the positive value required by positivity corresponds to the negative moment, like it was obtained by the QCD sum rule method. At the same time, the value of that moment, due to oscillations, was predicted to be an order of magnitude smaller than the value at this point. The minimal value of $\bar{g}_{2}$ for GS parametrization is about $10^{-3}$. This corresponds to a bound of the third moment from below of about $10^{-4}$, two orders of magnitude smaller than the QCD sum rule calculations.

One may note several possible reasons for such a discrepancy.

i) More accurate data may increase the degree of the twist-two positivity violation.

ii) The actual strength of the twist-three contribution may essentially exceed its lower positivity bound.

iii) The model [11] may be too crude. 
iiii) The (relatively) large value of twist-three contribution for $d$ quark is an ingredient of the QCD sum rule calculation rather than of the real nucleon structure. This point of view is supported by the fact that the recent SLAC data [16] seem to disagree with the QCD sum rule calculations.

Let us discuss this possibility in more details. The existence of a large twist-three contribution is due to the two sources - positivity constraint and large negative polarization. The positivity is likely to be present in sum rule calculations, as their main ingredients are spectral densities, corresponding to the on-shell particles in the intermediate states, which are in turn the main ingredients of the positivity proofs. At the same time, the $d$-quark negative polarization is introduced by local nucleon currents. As soon as QCD sum rules are unapplicable to describe the region of large and small $x$, and their ability to describe the $x$-dependence of parton distributions is rather limited, one can guess, that the $d$-quark polarization may be in fact overestimated in the region of moderate $x$, which is crucial to the positivity constraint. If so, this implicit overestimated polarization would be the actual source of the strong twist-three contribution, which should restore the validity of positivity constraints. One may speak about "positivity-driven" twist-three and it is a matter of future studies to decide, if it does reflect real physics or if it is just a consequence of the poor description of the $d$-quark longitudinal polarization.

The use of the new bound is resolving partially the puzzle, why the measured $A_{2}$ is such a small quantity. The fact, that the bound (1) is far from being saturated is obvious at low $x$ in the proton case, because, according to (4), it should be decreased by a factor $\sqrt{2}$, due to the small longitudinal asymmetry. The bound under consideration is even more useful with a negative longitudinal asymmetry, like in the neutron case. At the same time, the nucleon bounds are still undersaturated in comparison with the quark bounds, especially for $d$-quark, which may be saturated or even violated for some models. With the improved determination of the unpolarized $s(x)$ and $\bar{s}(x)$ distributions, positivity can be used to put a non trivial bound on the $\Delta s(x)$ and $\Delta \bar{s}(x)$. The general conclusion is that the bounds for each flavor are more sensitive, while their effects may be diluted for nucleons (especially, for proton).

We are indebted to E. Leader, E. de Rafael, A. Schäfer, A. Tkabladze and J.-M. Virey for useful discussions and correspondence and to Z-E. Meziani

${ }^{4}$ We are grateful to J.Ellis for this relevant comment 
and R. Windmolders for interest in the work. O.T. is thankful to Centre de Physique Théorique (CNRS-Luminy) for warm hospitality and to Université de Provence for financial support.

\section{References}

[1] N. Christ and T.D. Lee, Phys.Rev. 143, 1310 (1966).

[2] M.G. Doncel and E. de Rafael, Nuovo Cimento 4A, 363 (1971)

[3] J.Soffer and O.V.Teryaev, Phys.Lett. B419, 400 (1998)

[4] A.S.Gorsky and B.L.Ioffe, Particle World vol.1,114 (1990).

[5] J. Soffer, Phys. Rev. Lett. 74 (1995) 1292.

[6] O.V. Teryaev, Proceedings of 11th International Conference on Problems of Quantum Field Theory, Dubna, Russia, 13-17 Jul 1998: hep$\mathrm{ph} / 9808335$

[7] S.Wandzura and F.Wilczek, Phys.Lett. B82, 195 (1977)

[8] H.Georgi and H. D. Politzer, Phys.Rev. D14, 1829 (1976).

[9] M. Gluck, E. Reya, M. Stratmann and W. Vogelsang, Phys.Rev. D53, 4775 (1996).

[10] T. Gehrmann and W.J. Stirling, Phys.Rev. D53, 6100 (1996).

[11] O.V. Teryaev, Proceedings of the Workshop 'Spin Physics at HERA', DESY 95-200. p. 132-142.

[12] I.I. Balitsky, V.M. Braun and A.V. Kolesnichenko, Phys.Lett. B242, 245 (1990); Erratum: ibid., B308, 648 (1993)

[13] B. Ehrnsperger, A. Schäfer, W. Greiner and L. Mankiewicz, Phys.Lett. B343, 369 (1995);

[14] J. Soffer and O.V. Teryaev, Phys.Rev. D51, 25 (1995).

[15] A.V. Efremov, O.V. Teryaev and E Leader, Phys.Rev.D55, 4307 (1997). 
[16] P.L. Anthony et al., Phys.Lett. B458, 529 (1999). 